\title{
Bursting properties of units in cat globus pallidus and entopeduncular nucleus: the effect of excitotoxic striatal lesions
}

\author{
Robert N.S. Sachdev, Sid Gilman and J. Wayne Aldridge \\ Department of Neurology, University of Michigan, Ann Arbor, MI 48104 (U.S.A.)
}

(Accepted 11 December 1990)

Key words: Basal ganglia; Globus pallidus; Entopeduncular nucleus; $\gamma$-Aminobutyric acid; Burst discharge; Huntington's disease; Striatal lesion

\begin{abstract}
The bursting properties of units recorded in globus pallidus and entopeduncular nucleus were studied in awake cats sitting quietly before and after ipsilateral excitotoxic striatal lesions. A computerized statistical procedure was used to identify and evaluate bursts in the recorded spike trains. Bursts were assigned a quantitative statistical measure of burst 'strength' (or improbability) - the surprise value. Before the lesion, $34 \%$ of units in the globus pallidus and $60 \%$ of units in the entopeduncular nucleus exhibited bursts. Burst units had a significantly slower discharge rate and a significantly greater variability of discharge than non-burst units. The mean length of the interspike intervals immediately preceding the bursts was significantly longer than the overall median intervals in burst units. After the lesion, $21 \%$ of units in the globus pallidus and $11 \%$ of the units in the entopeduncular nucleus exhibited bursts. Burst units had significantly higher discharge rates and lower discharge variability after the lesion. In contrast, the lesion had no significant effect on the rate or variability of non-burst units. The differences between bursting and non-bursting units in discharge rate and variability disappeared after the lesion. In globus pallidus, the lesion resulted in a significant reduction in the mean number of bursts per unit, surprise value per burst, mean length of bursts, and number of spikes per burst, and a significant increase in the mean discharge rate of burst units. In entopeduncular nucleus, the small number of bursts recorded after the lesion precluded a useful statistical comparison of the effect of striatal lesions on the properties of the bursts. This study demonstrates that removing striatal projections to globus pallidus and entopeduncular nucleus decreases bursting in these nuclei, indicating that intact striatal projections are necessary for the normal production of bursts in these regions.
\end{abstract}

\section{INTRODUCTION}

Neuronal burst discharges are thought to be important in transmitting information in the nervous system ${ }^{19,35}$ and have been implicated in sleep ${ }^{23,24,25}$, epilepsy $y^{11,34,52,55}$, and long-term potentiation ${ }^{6,7}$. Bursting has been found in studies of single unit discharge in the globus pallidus (GP) and entopeduncular nucleus (EPN) of the $\mathrm{cat}^{28,36}$. Neurons in the striatum utilizing $\gamma$-aminobutyric acid (GABA) as a neurotransmitter project to GP and $\mathrm{EPN}^{27,43,46,48}$, and evidence suggests that the neurotransmitter GABA may be involved in neuronal bursting. Striatal stimulation or application of GABA to pallidal neurons inhibits GP neuronal discharge ${ }^{42,43}$. In addition, application of GABA on thalamic ${ }^{56,57}$, substantia nigra ${ }^{1}$ and hippocampal ${ }^{5,16,17}$ neurons reduces neuronal excitability and bursting. The application of GABA antagonists on hippocampal neurons increases burst discharges in these neurons ${ }^{34,52}$. Striatal lesions produced with the excitotoxin ibotenic acid result in a $90-95 \%$ decrease in extracellular GABA in striatal targets ${ }^{53}$. Thus, it might be expected that striatal lesions would increase bursting in GP and EPN. Excitotoxins, which destroy all classes of striatal neurons and leave the fibers of passage intact, are thought to produce neuropathological and neurochemical changes similar to those seen in Huntington's disease ${ }^{8}$. ${ }^{12,60}$. Thus, issues related to Huntington's disease can be addressed in studies of animals with excitotoxic striatal lesions.

In the present study we have examined the effects of excitotoxic lesions of the striatum on burst discharges in the GP and EPN. The present study presents additional analysis of data presented in a recently published report ${ }^{50}$. In this report, the effects of striatal lesions on tonic discharge properties of neurons in the pallidum were analyzed. This work indicated that the variability of interspike intervals in the GP and EPN decreased after striatal lesions. Alterations in the variability of discharge might be related to altered bursting properties of neurons in the pallidum. Thus, in the present study we examined the influence of striatal lesions on neuronal burst discharges in GP and EPN. 


\section{MATERIALS AND METHODS}

\section{Procedures}

Four cats were habituated to the recording procedures and trained to sit quietly in a loose restraint. Two to three weeks later, they were prepared surgically for chronic unit recordings. In each animal, a chamber was positioned stereotaxically and fixed to the left side of the cranium. The chamber provided access to the GP, EPN, caudate nucleus and putamen. One week after surgery, the animals were rehabituated to the recording apparatus and exploratory recordings were initiated to locate the pallidum and striatum. Data collection with tungsten microelectrodes began 2-3 weeks after surgery. Computerized procedures for digitizing spikes on-line assisted with the recording ${ }^{3}$. Units recorded at each site were discriminated from each other and from background noise on the basis of their negative peak amplitudes, positive peak amplitudes, and peak-to-peak times $^{59}$. Discriminated units were used in subsequent analyses. The results presented in this paper represent additional analysis of data presented in a recently published paper ${ }^{50}$

Spontaneous unit activity was recorded from the GP and EPN of alert cats sitting quietly. The animals were loosely restrained with a plastic collar. The behavioral state of the cats was monitored by EEGs, movement detectors (accelerometers or high-impedance cables), video monitors, or a combination of these. Unit activity occurring during movement or $500-1500 \mathrm{~ms}$ before or after a movement was discarded. In the first two cats, EEG activity was recorded to identify slow wave, large amplitude signals indicating early sleep stages. Since the cats were usually awake during the short (less than $2 \mathrm{~h}$ ) recording sessions and since visual observations proved as useful as EEGs, the subsequent two cats were monitored with a video camera alone. The first two animals were studied both before and after the lesion. In these cats, penetrations through GP and EPN were made in a checkerboard fashion. A set of recording tracks through the GP and EPN was studied before the lesion. The remaining tracks neighboring those studied before the lesion were examined after the lesion. This procedure of recording from neighboring tracks before and after the lesion ensures that unit data are gathered from similar sites. One of the remaining two cats was studied only before the lesion, and the other was studied only after the lesion. This procedure of studying one cat only after the lesion provided some control for the effects of electrode damage on the discharge in GP and EPN. Damage caused by the electrode had no noticeable effect on the discharge pattern in GP and EPN, as pre-lesion and post-lesion data obtained from animals studied both before and after the lesion were similar to that obtained from animals studied only before or after the lesion. Unilateral striatal lesions were produced by multiple injections of ibotenic acid (2 $\mu \mathrm{g} / 200 \mu \mathrm{l})$ into the caudate nucleus and putamen. Eleven to 19 injection sites ranging from 0.25 to $1.5 \mu \mathrm{l} / \mathrm{site}$ were used to produce the lesions. Cell loss in the striatum is thought to occur within a week after injection of the excitotoxin ${ }^{13}$. Thus, in the present study unit activity in the pallidum was recorded between 7 and 160 days after the lesion.

Histologic reconstructions of the recording sites were facilitated by marking 6 recording tracks with deposits of iron on the final day of recording. Direct current $(100 \mu \mathrm{A}$ for $30-45 \mathrm{~s})$ was passed through a bipolar steel electrode, providing iron deposits that were stained blue by adding $1.5 \%$ potassium ferrocyanide to the $10 \%$ formalin perfusing solution. The animals were perfused transcardially under deep barbiturate anesthesia. The brains were removed, postfixed in sucrose/formalin solution, frozen, sectioned, and stained with Cresyl violet. The recording tracks and lesions were reconstructed and drawn on maps traced from stained sections. The lesion size was determined with the aid of an image analysis computer. An outline of the striatum was traced on a digitized image of representative coronal sections. The striatal areas on the side of the lesion and on the contralateral side were computed and compared.

\section{Data analysis}

Conventional techniques such as the autocorrelation method or examination of interval distributions cannot detect individual bursts in a spiketrain. Consequently, procedures similar to those described by Legendy and Salcman were used to detect bursts ${ }^{35}$. A group of spikes $(n>3)$ was initially identified as a burst if the mean rate of activity for the group of spikes was at least twice that of the mean rate for the whole spiketrain. Bursts were assigned a quantitative, statistical measure of burst strength - the Poisson surprise value defined as the negative logarithm of the probability of the occurrence of a burst in a random (Poisson) spiketrain. Following the initial calculation of the surprise value, this value was maximized by adding an additional spike to the end of the burst to determine whether this would increase the surprise value. If adding the spike increased the surprise value, the spike was included in the burst. This process of adding spikes if they increased the surprise value was repeated as long as the surprise value increased. Maximization of the surprise value was pursued further by removing spikes from the beginning of the burst. If removing a spike increased the surprise value, the spike was removed from the burst. The surprise value for each burst, the length of each burst, the number of spikes in each burst, the rate within each burst, and the number of bursts per 1000 spikes (burst rate) were calculated. A group of spikes was considered to be a burst if the Poisson surprise value was greater than 3 , indicating that the particular group of spikes had a less than 1 in 1000 chance of being a random event. To determine the length of the interval preceding the burst, the burst and the interval preceding the burst were displayed graphically. The intervals were then directly measured from the print-out. For long pre-burst intervals $(400-2000 \mathrm{~ms})$, the actual interval could exceed the displayed value. Nevertheless, in these cases the displayed value was used as an estimate of the interval. This method tends to underestimate the length of the long pre-burst intervals. The median and coefficient of variation (standard deviation/mean) of the interspike intervals for all units were also calculated.

The Mann-Whitney $U$-test and the chi-square test were used to assess the significance of changes in bursting. The binomial test was
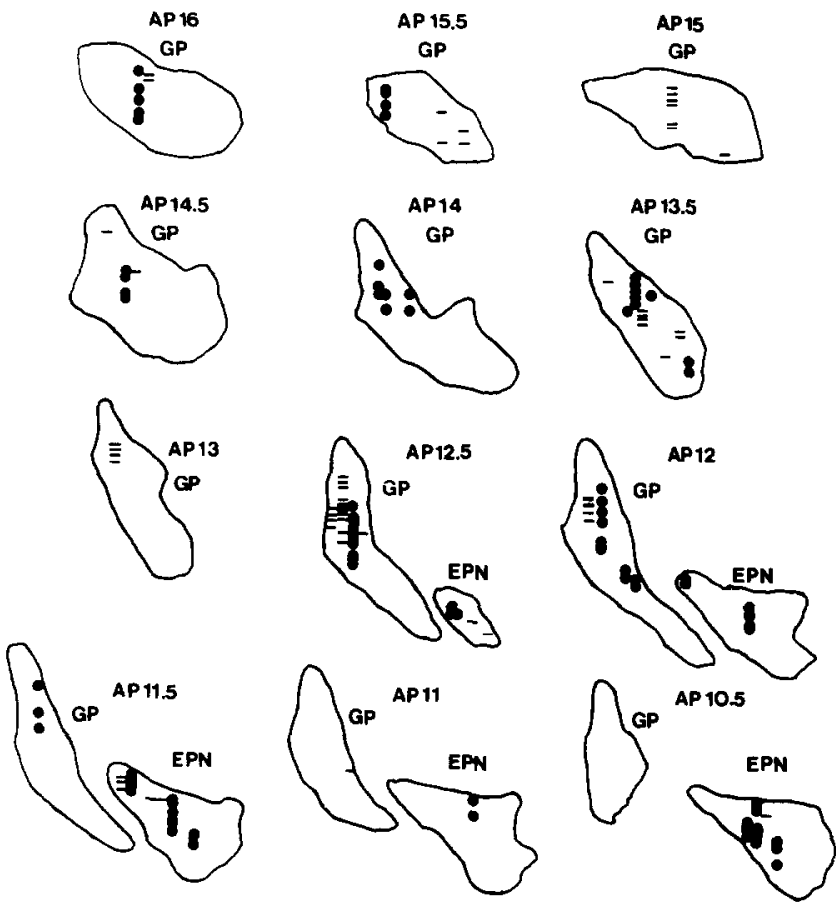

Fig. 1. Representation of recording sites where bursts were detected. Pre-lesion recording sites from all animals are plotted.as filled circles and post-lesion recording sites from all animals are plotted as dashes. Units exhibiting no bursts were interspersed with the units exhibiting bursts. 


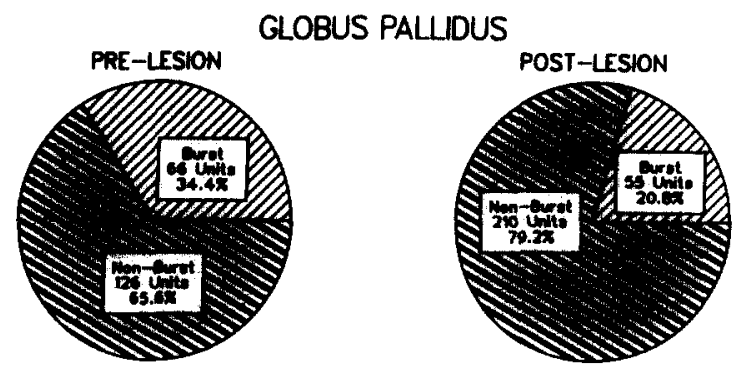

ENTOPEDUNCULAR NUCLEUS
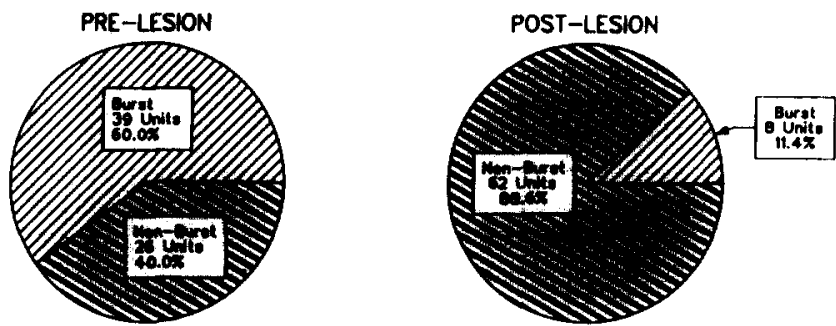

Fig. 2. Proportion of units that burst in globus pallidus (top) and entopeduncular nucleus (bottom). The proportion of units that burst decreases in both structures after the lesion.

used to assess the significance of the difference between the median interval of the unit and the length of the interval immediately preceding the burst. To determine whether the surprise value was linearly correlated to any of the other properties of a burst, correlation coefficients were also calculated.
TABLE 1

Discharge properties of units in globus pallidus

Numbers in brackets represent standard errors.

\begin{tabular}{|c|c|c|c|c|}
\hline & \multicolumn{2}{|l|}{ Pre-lesion } & \multicolumn{2}{|c|}{ Post-lesion } \\
\hline & Bursting & Non-burst & Bursting & Non-burst \\
\hline $\begin{array}{l}\text { Median inter- } \\
\text { val (ms) }\end{array}$ & $37(3.3)$ & $35(2.1)$ & $25(3.1)$ & $33(2.5)$ \\
\hline $\begin{array}{l}\text { Mean inter- } \\
\quad \text { val (ms) }\end{array}$ & $94(12)$ & $45(4.6)^{*}$ & $46(6.7)$ & $42(2.9)$ \\
\hline $\begin{array}{l}\text { Coefficient } \\
\text { of variation }\end{array}$ & $2.1(0.2)$ & $0.9(0.02)^{*}$ & $1.4(0.1)$ & $0.9(0.03)$ \\
\hline $\begin{array}{l}\text { Number of } \\
\text { units }\end{array}$ & 66 & 126 & 55 & 210 \\
\hline
\end{tabular}

\section{RESULTS}

\section{Striatal lesion}

The striatal lesion extended through the entire rostrocaudal extent of the caudate nucleus in all animals. The rostral putamen was also lesioned in all cats. In each section examined, the lesion resulted in a $25-50 \%$ loss of striatal tissue on the lesioned side as compared to the contralateral side ${ }^{50}$. The lesion produced no noticeable
PRE-LESION
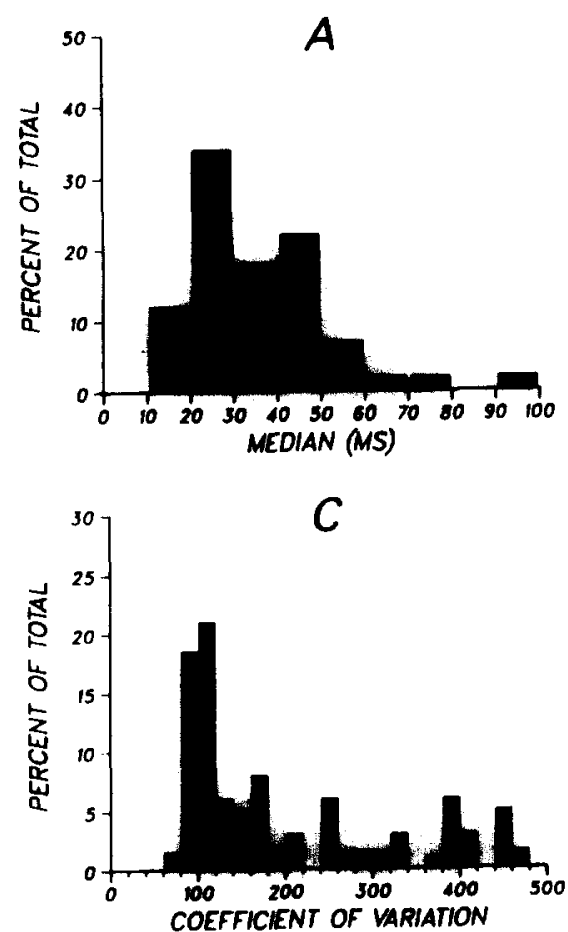

POST-LESION
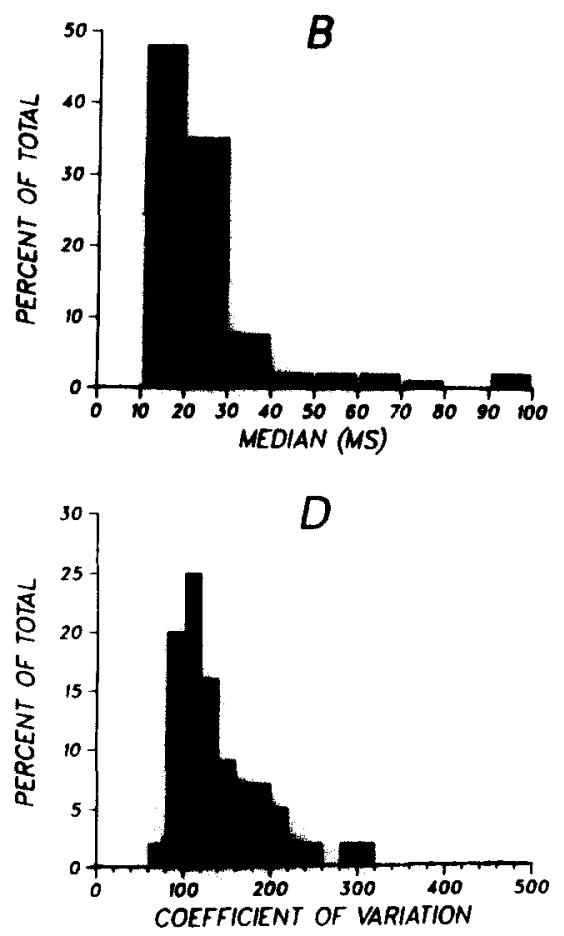

Fig. 3. Distribution of median interspike intervals in globus pallidus before (A) and after (B) striatal lesion. Distribution of coefficients of variation of the interspike intervals before $(C)$ and after $(D)$ striatal lesion. The distributions of the median interspike intervals and coefficients of variation include only units exhibiting bursts. 
behavioral, motor, or postural changes in any animal.

\section{Site of recording}

Burst units in the GP and EPN (Fig. 1) were detected in similar regions before and after the lesion. Burst units were intermingled with non-burst units.

\section{Globus pallidus}

Prior to the lesion, one third of the units in GP (66 of 192) exhibited bursts (Fig. 2). On average, both burst units and non-burst units had similar median interspike intervals, however, the mean interspike intervals of burst units were significantly longer than those of non-burst units (Table I). Burst units had significantly greater variability of discharge than non-burst units, i.e. burst units had larger coefficients of variation than non-burst units (Table I).

The striatal lesion had no effect on the overall discharge rate or variability of non-burst units (Table I), but the lesion had substantial effects on burst units. The lesion resulted in a $14 \%$ decrease in the proportion of units exhibiting bursts $(P<0.01$, chi-square test $)$; after the lesion only 55 of 265 units exhibited bursts (Fig. 2). Prior to the lesion, burst units had significantly longer median intervals and significantly greater variability than burst units after the lesion $(P<0.0001$, Mann-Whitney $U$-test). The lesion resulted in a $32 \%$ decrease in the length of the median interval and a $33 \%$ decrease in the coefficient of variation (Table I). Examination of the distribution of median intervals and coefficients of

\section{TABLE II}

Properties of bursts in globus pallidus and entopeduncular nucleus

Numbers in brackets represent standard errors.

\begin{tabular}{|c|c|c|c|c|}
\hline & \multicolumn{2}{|c|}{ Globus pallidus } & \multicolumn{2}{|c|}{$\begin{array}{l}\text { Entopeduncular } \\
\text { nucleus }\end{array}$} \\
\hline & Contral & Lesion & Control & Lesion \\
\hline $\begin{array}{l}\text { No. of spikes } \\
\text { Intra-burst rate }\end{array}$ & $14(1.9)$ & $8(0.4)^{*}$ & $9(0.4)$ & $7(0.4)$ \\
\hline $\begin{array}{l}(\mathrm{hz})^{\mathrm{a}} \\
\text { Burst duration }\end{array}$ & $183(35)$ & $251(23)^{*}$ & $258(32)$ & - \\
\hline (ms) & $200(30.5)$ & $43(5.6)^{*}$ & $60(12.5)$ & $45(15)$ \\
\hline Surprise value & $8(0.8)$ & $4(0.13)^{*}$ & $5(0.1)$ & $5(1.3)$ \\
\hline Bursts/1000 spikes & $14(2.2)$ & $11(2.6)$ & $13(2.6)$ & - \\
\hline No. of bursts & 273 & 158 & 202 & 8 \\
\hline No. of units & 55 & 65 & 39 & 6 \\
\hline
\end{tabular}

${ }^{a}$ Computing an intra-burst rate from the mean values of the number of spikes and burst duration give different results for intra-burst rate than the mean computed from individual bursts (shown). The former reduces the impact of the short bursts with few spikes, especially when these bursts have a high intra-burst rate.

* $P<0.0001$ as assessed by the Mann-Whitney $U$-test.

$\mathrm{ms}=$ milliseconds, $\mathrm{hz}=$ spikes $/ \mathrm{s}$. variation suggests that the proportion of burst units with long median intervals (Fig. 3A,B) or large coefficients of variation (Fig. 3C,D) decreased considerably. Concomitant to the decreased variability, the rate of bursting (bursts/1000 spikes) in neurons exhibiting bursts also decreased by $23 \%$ after the lesion (Table II).

The striatal lesion altered the characteristics of the bursts (Fig. 4). The mean burst length was significantly reduced (Table II), as demonstrated by a decreased proportion of bursts greater than $80 \mathrm{~ms}$ in duration (Fig. $5 A, B)$. The mean number of spikes per burst was

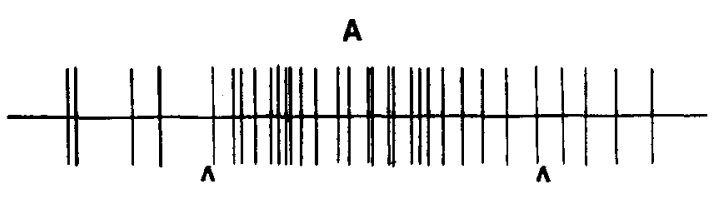

B

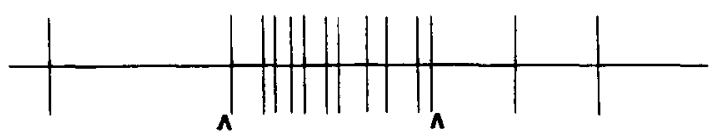

C

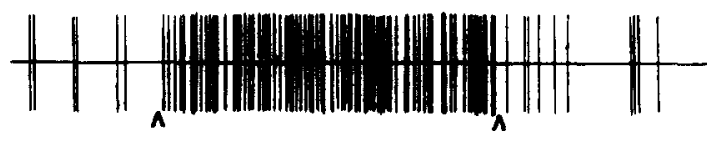

D

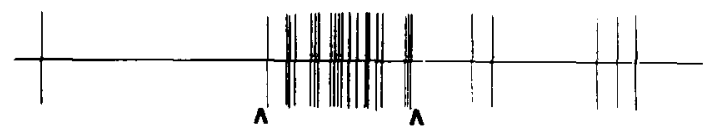

E
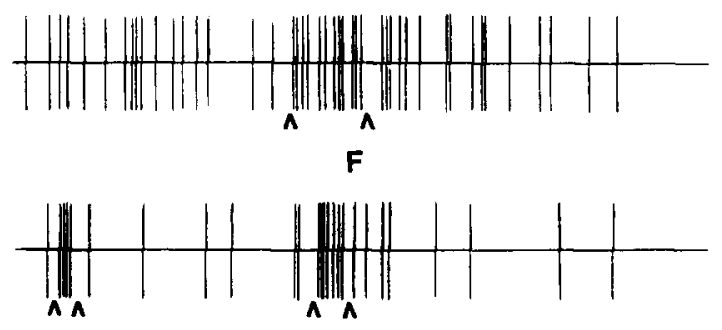

G

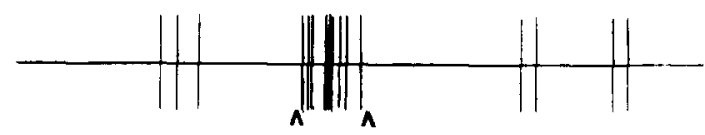

H

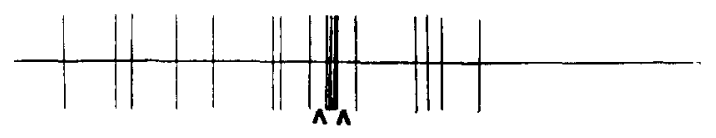

$100 \mathrm{~ms}$

Fig. 4. Examples of bursts in GP and EPN. Pre-lesion GP (A-D), pre-lesion EPN ( $E$ and $F$ ) and post-lesion $G P$ ( $G$ and $H$ ). Arrowheads mark the beginning and end of bursts. Bursts recorded after the lesion are shorter and contain fewer spikes than bursts recorded before the lesion. In trace $\mathrm{F}$, there are two bursts. 
reduced by $43 \%$ (Table II). The distributions of the number of spikes in bursts demonstrate that, after the lesion, the proportion of bursts containing many spikes ( $n$ $>10$ ) decreased (Fig. 5C,D). The lesion also resulted in a significant increase in the rate of discharge within bursts (Table II), and the distributions of the rates suggest that the proportion of bursts with a high discharge rate increased after the lesion (Fig. 5E,F). The average surprise value, an indication of burst strength (or burst improbability), decreased significantly after the lesion (Table II). This was most evident from the decreased proportion of bursts with large surprise values (Fig. $6 \mathrm{~A}, \mathrm{~B})$. The decreased surprise value was probably related to the decreased number of spikes per burst since the correlation between the surprise value and the number of spikes in the burst was so strong (correlation coefficient, $r=0.95)$. The surprise value was not as well correlated to the length of the burst $(r=-0.4)$, the rate of discharge in the burst $(r=-0.1)$, the mean interval length $(r=0.1)$, or the median interval length $(r=-0.1)$ of the units (Fig. 7). The intra-burst rate was weakly related to the overall discharge rate $(r=0.5)$.

GP bursts were frequently preceded by long intervals (Fig. 4). These intervals were significantly longer than the median interval for the unit $(P<0.01$, binomial test $)$. The mean pre-burst interval was $99 \mathrm{~ms}$ (S.E.M. = 16), about 3 times longer than the average median interval. Following the lesion the pre-burst interval decreased significantly to $78 \mathrm{~ms}$ (S.E.M. $=8.9)(P<0.005$. Mann-Whitney $U$-test). Even after the lesion, however, the pre-burst intervals were significantly (3 times) longer than the median interval $(P<0.01$, binomial test $)$.

\section{PRE-LESION}
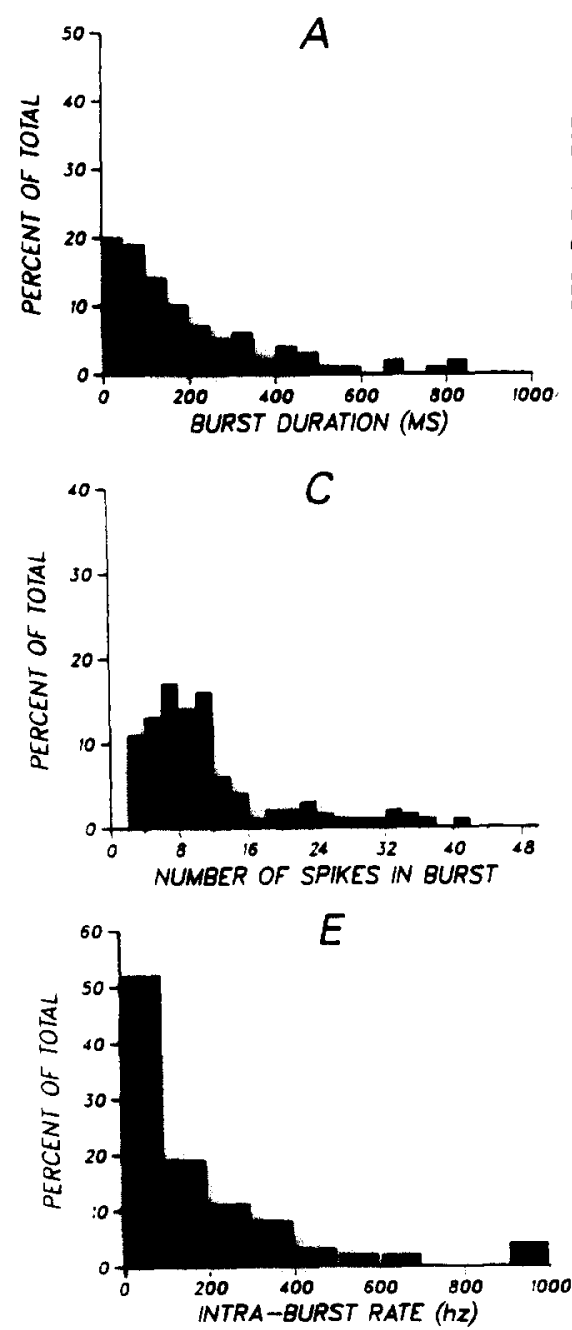

POST-LESION
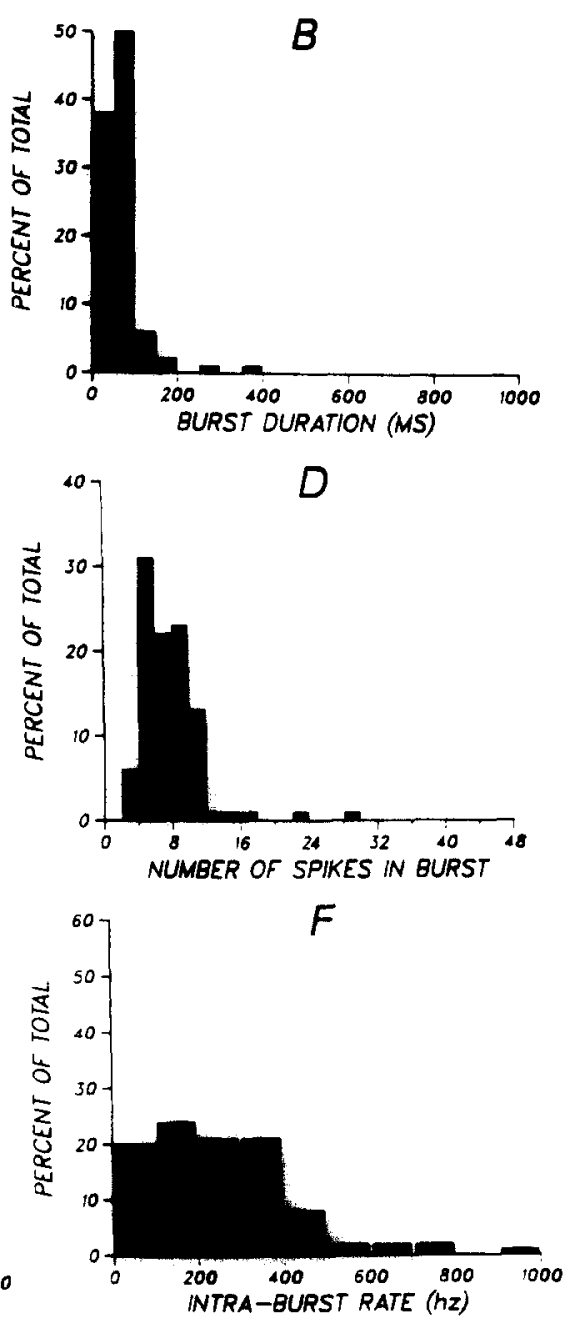

Fig. 5. Distribution of burst duration, number of spikes, and surprise values in globus pallidus bursts before and after the striatal lesion. Burst duration pre-lesion (A) and post-lesion (B). Number of spikes in the burst pre-lesion (C) and post-lesion (D). Intra-burst rate pre-lesion (E) and post-lesion $(F)$. Note that the post-lesion distributions of burst duration and number of spikes shift towards shorter values whereas the post-lesion distribution of rates shifts towards larger values. 
PRE-LESION

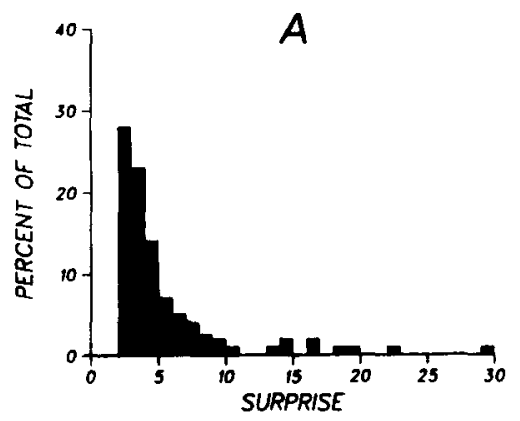

POST-LESION

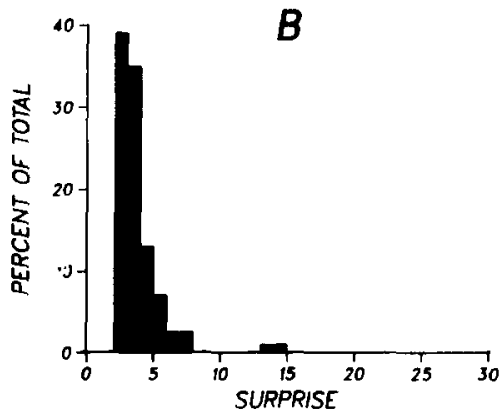

Fig. 6. Distribution of surprise values in globus pallidus pre-lesion (A) and post-lesion (B).

\section{Entopeduncular nucleus}

Prior to the lesion, a majority $(60 \%)$ of units exhibited bursts (Fig. 2). On average, the interspike intervals of burst units were significantly more variable $(P<0.001$, Mann-Whitney $U$-test) than non-burst units (Table III). Burst units also had significantly lower mean rates of discharge $(P<0.001$, Mann-Whitney $U$-test) than nonburst units (Table III). Following the striatal lesion the proportion of units exhibiting bursts decreased markedly $(P<0.01$, chi-square test), to only $11 \%$ (Fig. 2). The small number of bursts recorded after the lesion precluded a useful statistical comparison of the effect of the striatal lesion on the properties of bursts in EPN. As in GP, however, the lesion did not affect the overall discharge properties of non-burst units (Table III).

Prelesion comparison of globus pallidus and entopeduncular nucleus

Prior to the lesion, a larger proportion of burst units in EPN had small median intervals $(P<0.001$, Mann-
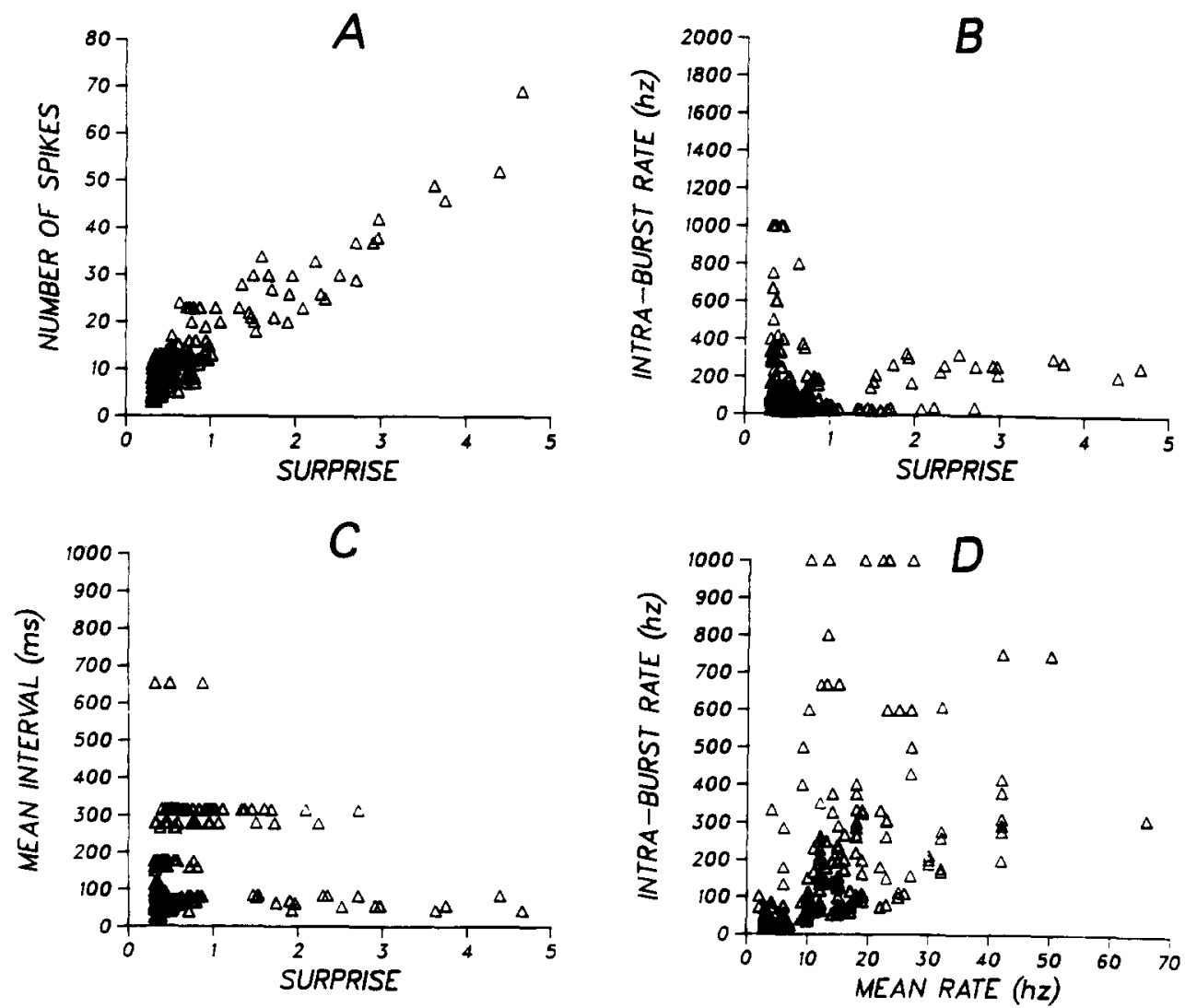

Fig. 7. Scatter plots of pre-lesion globus pallidus burst parameters: A: number of spikes in burst vs. surprise; B: intra-burst rate vs. surprise; C: mean interval vs. surprise; and D: intra-burst rate vs. overall mean rate. The values illustrated in the scatter plots are culled from 175 globus pallidus bursts. The surprise values are linearly related to the number of spikes in the burst. 

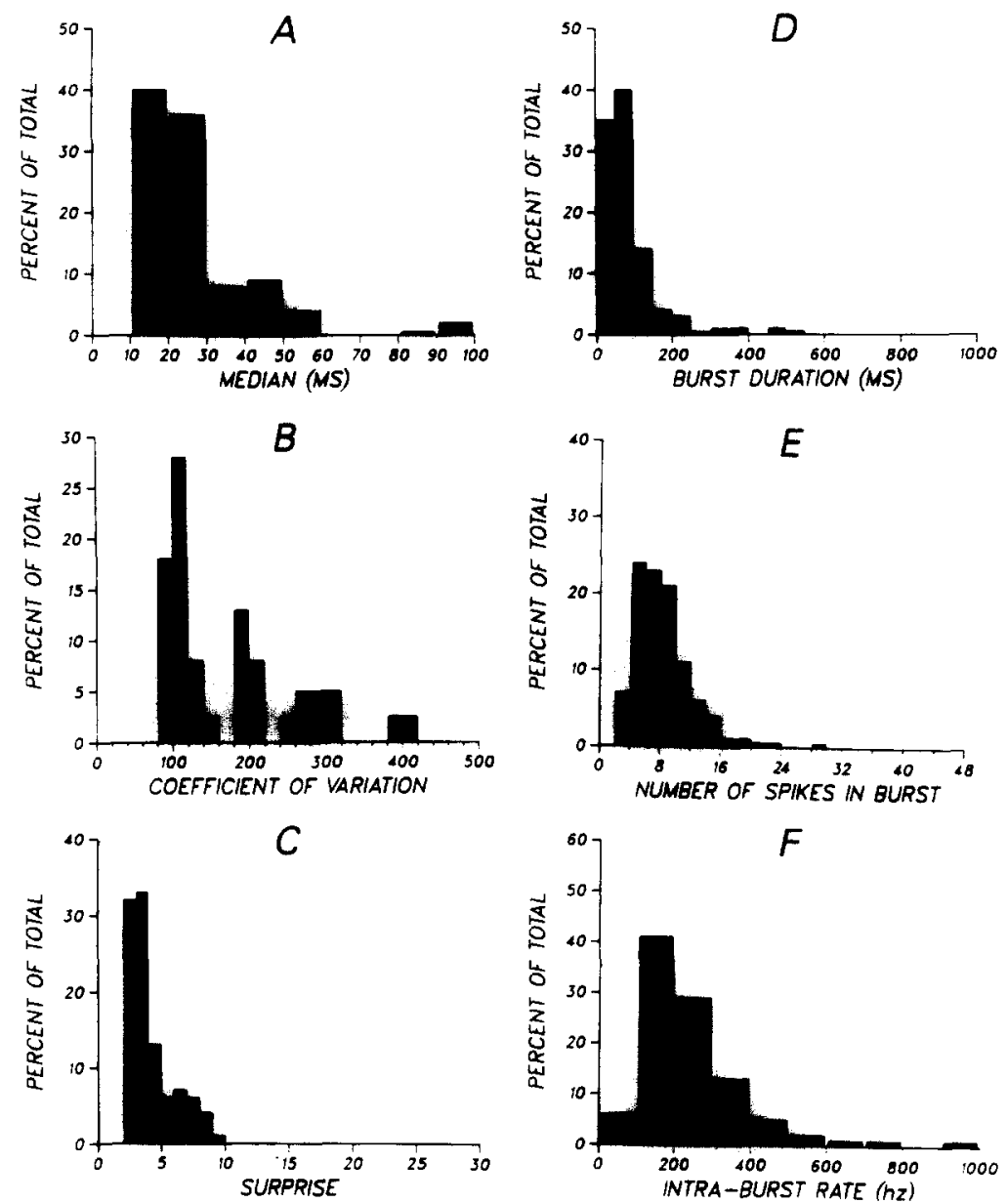

Fig. 8. Distributions of burst parameters in entopeduncular nucleus: A: median interspike intervals for units exhibiting bursts; B: coefficients of variation of the interspike intervals for units exhibiting bursts; C: surprise; D: burst duration; E: number of spikes in burst; and F: intra-burst rate.

Whitney $U$-test) as compared to GP (compare distributions in Fig. 8A and Fig. 3A). Burst units in EPN were also less variable $(P<0.0001$, Mann-Whitney $U$-test $)$ than burst units in GP (Fig. 8B vs. Fig. 3B). Bursts in

\section{TABLE III}

\section{Discharge properties of units in entopeduncular nucleus}

Numbers in brackets represent standard errors.

\begin{tabular}{|c|c|c|c|c|}
\hline & \multicolumn{2}{|l|}{ Pre-lesion } & \multicolumn{2}{|c|}{ Post-lesion } \\
\hline & Bursting & Non-burst & Bursting & Non-burst \\
\hline $\begin{array}{l}\text { Median inter- } \\
\text { val (ms) }\end{array}$ & $34(3.3)$ & $32(5.9)$ & $48(14.5)$ & $32(2.5)$ \\
\hline $\begin{array}{l}\text { Mean inter- } \\
\text { val (ms) }\end{array}$ & $60(8.3)$ & $47(6.2)^{*}$ & $61(18.3)$ & $40(4.8)$ \\
\hline $\begin{array}{l}\text { Coefficient } \\
\text { of variation }\end{array}$ & $1.8(0.1)$ & $0.9(0.2)^{*}$ & $0.8(0.1)$ & $0.7(0.03)$ \\
\hline $\begin{array}{l}\text { Number of } \\
\text { units }\end{array}$ & 39 & 26 & 8 & 62 \\
\hline
\end{tabular}

$P<0.001$ as assessed by the Mann-Whitney $U$-test. $\mathrm{ms}=$ milliseconds.
EPN had significantly smaller mean surprise values $(P<$ 0.005 , Mann-Whitney $U$-test) than bursts in GP (Table I). Bursts with small surprise values (surprise $<5$ ) were more common in EPN than in GP (Fig. 8C vs. Fig. $5 \mathrm{E}$ ). Before the lesion, bursts in EPN on the average were $70 \%$ shorter $(P<0.0001$, Mann-Whitney $U$-test $)$, contained 35\% fewer spikes $(P<0.001$, Mann-Whitney $U$-test), and had a $27 \%$ higher rate of discharge within the burst $(P<0.0001$, Mann-Whitney $U$-test $)$ than bursts in GP (Fig. 8D,E,F). In EPN the pre-burst interval was $91 \mathrm{~ms}($ S.E.M. = 9) and, as in GP, the pre-burst interval in EPN was more than 3 times the median interval length $(P<0.01$, binomial test $)$.

\section{DISCUSSION}

In this study, we found that excitotoxic lesions of the striatum resulted in decreased neuronal bursting in GP and EPN. In GP, the lesions resulted in a decreased proportion of units exhibiting bursts and in a higher overall discharge rate and lower variability in burst units. 
In EPN, the striatal lesions resulted in a dramatic decrease in the proportion of units that burst. The lesions had no effect on the properties of non-burst units in either GP or EPN.

In GP, the reduction in bursting following the striatal lesion was accompanied by an increased intra-burst discharge rate, decreased burst length, fewer spikes per burst and decreased surprise values of the bursts. A decreased overall mean interval length (i.e. an increase in the discharge rate) in burst units and an increased rate within the burst are both consistent with the loss of a tonic inhibitory input from the striatum. The decreased variability in post-lesion spiketrains is probably related to a reduced number of bursts. The decreased surprise value, decreased length of bursts, and decreased number of spikes per burst all suggest that GP bursts are 'weaker' after the lesion. These results indicate that striatal inputs are important for production of 'normal' bursts in GP and EPN. It may be that partially deafferented GP and EPN neurons are unable to produce 'normal' bursts. Alternately, these changes in bursting may reflect a compensatory response to the lesion.

The increased post-lesion mean discharge rate in GP could potentially affect the detection of bursts in GP. The detection of bursts with the surprise technique depended on the mean discharge rate of individual spiketrains. Initially, a group of 3 spikes in a spiketrain was considered to be a burst if that group had a mean discharge rate that was at least twice the mean discharge rate for the spiketrain. Thus, the increased post-lesion discharge rate in GP might by itself reduce the number of bursts detected in GP after the lesion. To be classified as a burst, however, that group of 3 spikes also had to have a surprise value of at least 3 , which usually meant an addition of spikes. The number of spikes per burst usually exceeded 3 . Thus, the direct relationship between the mean discharge rate and the properties of bursts was weakened. Data from EPN indicate that the reduction in the number of bursts detected in EPN occurs independently of any changes in the mean discharge rate. The number of bursts detected in EPN decreased after the lesion even though the mean discharge rate was not affected by the lesion. Moreover, the huge difference between the mean discharge rate and the mean intraburst rate in GP suggests that post-lesion alterations in the mean discharge rate have little effect on the detection of bursts. The pre-lesion intraburst rate in GP is 17 times the mean discharge rate in GP. This difference in the pre-lesion intra-burst rate and the mean rate is much larger than the difference between the pre-lesion and post-lesion mean rates.

Legendy and Salcman developed the 'Poisson surprise' procedure for detecting and evaluating bursts in the visual cortex of alert cats ${ }^{35}$. They used a surprise value of 10 as a cut-off for selecting bursts. Bursting in cat GP and EPN is different from bursting in the visual cortex ${ }^{35}$. Spike trains in the visual cortex exhibit more bursts and have slower rates of discharge than those in the pallidum. The burst discharges in visual cortex have on average larger surprise values than bursts in GP and EPN. The mean discharge rate in visual cortex bursts is on average only 3-6 times higher than the overall mean discharge rate whereas the mean discharge rate in GP and EPN bursts is on average 7-10 times the overall median discharge rate. The surprise cut-off in the present study was 3, which might result in the inclusion of less intense bursts in our sample as compared with the sample from visual cortex. It is possible that the differences in bursting reflect differences in inputs, receptors, and transmitters found in these two regions ${ }^{20,29,37}$. For example, the cat pallidum probably receives only one major glutamatergic input $^{54}$ and has few glutamate receptors ${ }^{29,37}$, whereas the visual cortex receives many glutamatergic inputs and has a large number of glutamate receptors ${ }^{20,29,37}$. Glutamate is an excitatory transmitter thought to produce burst firing ${ }^{55}$.

In the present study, we excluded data associated with limb and body movements because these movements are thought to alter the pattern of unit activity in the basal ganglia $^{2,21,22}$. Our goal was to study spontaneous discharge with a constant behavioral baseline. To this end, we trained the animals to sit quietly, and monitored their movements to exclude any data collected during movements. In contrast, the goal in the work of Legendy and Salcman was to develop a technique for evaluating bursts, therefore, they did not restrict visual stimulation or eye movements, which are thought to trigger burst discharges in the visual cortex. In fact, in Legendy and Salcman's study, visual stimulation elicited most of the burst discharges. This fundamental difference in the recording paradigm and goals of the two studies may be yet another reason for the observed differences in bursting ${ }^{35}$.

Previous studies of unit activity in the cat pallidum identified burst discharges in both GP and $\mathrm{EPN}^{28,36}$. Previous investigations in monkey and the present study in cat demonstrate that a significant proportion of units in the lateral GP of monkey and in the GP of cat exhibit bursts and burst units in both cat and monkey have much slower mean rates of discharge than non-burst units ${ }^{21}$, ${ }^{22,26}$. No bursts have been described in units of the medial GP of monkey. In the present study, however, we find that the EPN has burst units. Besides species differences, methodologic differences may account for the results. Earlier studies did not have the advantages of the surprise technique, which provides detailed information 
about the characteristics of individual bursts. In the earlier studies, bursting was assessed by examining interval distribution characteristics, i.e. mode, and the mean rate of discharge. Interval distribution characteristics and even autocorrelation measures provide no information about individual bursts in a spiketrain.

Our work suggests that units in GP have different bursting characteristics than those in EPN. GP bursts are more prominent than EPN bursts, i.e. they are longer, contain more spikes, and have larger surprise values. Consequently, bursts in GP are more noticeable than bursts in EPN, and this may account for the absence of bursting in the earlier descriptions of unit discharge in the monkey medial globus pallidus. The different peptidergic inputs from striatum to GP and EPN may explain the differences between the bursts that normally occur in GP and EPN and may also explain why bursting in EPN is reduced compared to GP. Enkephalin, the peptidergic transmitter co-localized with GABA in striatal inputs to $\mathrm{GP}^{4,30,46}$, is thought to be inhibitory ${ }^{40.41}$ whereas substance $\mathbf{P}$, the peptidergic transmitter found in the striatal inputs to the $\mathrm{EPN}^{30,47}$, is thought to produce long slow depolarizations conducive for bursting ${ }^{18.47}$. The larger proportion of bursting units detected in EPN as compared to GP suggests that substance P may have a role in burst production.

Decreased bursting in GP and EPN after the striatal lesion is not consistent with our initial prediction, which was based on the findings that (1) GABA application reduces neuronal excitability and bursting ${ }^{16.17 .23 .56 .57}$, and (2) decreased extracellular GABA in GP and EPN results from excitotoxic striatal lesions ${ }^{53}$. On the contrary, our results suggest that striatal input is necessary for the normal production of bursts in GP and EPN. The idea that GABA is responsible for bursts in these structures is supported by the observation that the pre-burst interval in GP and EPN is usually longer than the median interval for the unit, suggesting that GABAergic inhibition results in the long interval preceding a burst. The long inhibition may then be followed by rebound bursting in GP and EPN, similar to that seen in thalamus ${ }^{23.32 .56 .57}$. Even though the GABA-mediated mechanism of rebound bursting is consistent with our

\section{REFERENCES}

1 Aghajanian, G.K. and Bunney, B.S., Dopamine 'autoreceptors': pharmacological characterization by microiontophoretic single cell recording studies, Naunyn-Schmied. Arch. Pharmacol., 297 (1977) 1-7.

2 Aldridge, J.W., Anderson, R.J. and Murphy, J.T., Sensorymotor processing in the caudate nucleus and globus pallidus: a single-unit study in behaving primates,, Brain Research, 192 (1980) 3-16.

3 Aldridge, J.W., Walden, J.L. and Gilman, S., Enhancing high results, other GABAergic mechanisms may be important in bursting. It may be that, as in the thalamus, the resting membrane potential of a neuron determines whether a neuron bursts ${ }^{32}$. The more hyperpolarized the resting membrane potential of a thalamic neuron, the more likely it is to burst when depolarized ${ }^{32}$. A similar mechanism may work in GP and EPN. Perhaps, following the striatal lesion, the decreased amount of GABA in GP and EPN results in a chronically more depolarized resting membrane potential, thereby reducing bursting.

Another mechanism may also be important in explaining decreased bursting in GP and EPN after striatal lesion. Recent work in the hippocampus suggests that a reduction in inhibition by itself is not sufficient for increased bursting ${ }^{11}$. In addition to reduced inhibition, excitatory inputs must be active for bursting to occur. In our study, it is likely that the excitatory inputs to GP and EPN neurons were affected by the striatal lesion. The majority of excitatory inputs to GP and EPN are from the subthalamic nucleus ${ }^{9,10.38 .39}$, and this nucleus in turn receives inhibitory inputs from $\mathrm{GP}^{33}$. Our work demonstrates that striatal lesions increase the discharge rate in $\mathrm{GP}^{50}$. This increase presumably enhances inhibitory input to the subthalamic nucleus, probably causing subthalamic neurons to discharge more slowly. This would reduce the number of EPSPs impinging on GP and EPN neurons, and would reduce bursting in these nuclei even though the amount of GABAergic inhibition is decreased in both nuclei.

Hypotheses attempting to explain the mechanisms underlying the chorea $a^{14.15 .44 .45 .49}$ or bradykinesia ${ }^{58}$ of Huntington's disease have focused on the effects of striatal lesions on discharge rates in pallidum, VA/VL thalamus and cortex. Our results suggest that changes in burst discharges may also be important.

Acknowledgements. The authors gratefully acknowledge the technical assistance provided by D. Jaeger and J. Walden. The authors thank Drs. R. Albin, R.L. Macdonald, S. Winans Newman, R. Twyman and A.B. Young for their helpful suggestions. This work was supported in part by NIH grants NS-19613, and NS-07222, a grant from the Hereditary Disease Foundation, two fellowships awarded by the University of Michigan Medical School (to R.N.S.S.), and a grant from the United Cerebral Palsy Research and Education Foundation, Inc.

speed digitization of single unit neuronal activity on a microcomputer using a hybrid software-hardware technique, J. Neurosci. Methods, 28 (1989) 205-208.

4 Aronin, N., DiFiglia, M., Graveland, G.A., Schwartz, W.J. and Wu, J., Localization of immunoreactive enkephalins in GABA synthesizing neurons of the rat neostriatum, Brain Research, 300 (1984) 376-380.

5 Biscoe, T.J. and Straughan, D.W., Micro-electrophoretic studies of neurons in the cat hippocampus. J. Physiol. (London), 183 (1966) 341-359.

6 Bliss, T.V.P. and Lomo, T., Long-lasting potentiation of synaptic 
transmission in dentate area of anesthetized rabbit following stimulation of perforant path, J. Physiol. (London), 232 (1973) 357-374.

7 Brown, T.H., Chapman, P.F., Kairiss, E.W. and Keenan, C.L., Long-term synaptic potentiation, Science, 242 (1988) 724-728.

8 Bruyn, G.W., Huntington's chorea: historical, clinical and laboratory synopsis. In P. Vinken and G.W. Bruyn (Eds.), Handbook of Clinical Neurology, Vol. 6, North-Holland Publishing Co., Amsterdam, 1968, pp. 298-378.

9 Carpenter, M.B., Batton III, R.R., Carleton, S.C. and Keller, J.T., Interconnections and organization of pallidal and subthalamic nucleus neurons in the monkey, J. Comp. Neurol., 197 (1981) 579-603.

10 Carpenter, M.B., Carleton, S.C., Keller, J.T. and Conte, P., Connections of the subthalamic nucleus in the monkey, Brain Research, 224 (1981) 1-29.

11 Chamberlin, N.L. and Dingledine, R., GABAergic inhibition and the induction of spontaneous epileptiform activity by low chloride and high potassium in the hippocampal slice, Brain Research, 445 (1988) 12-18.

12 Coyle, J.T. and Schwarcz, R., Lesion of striatal neurons with kainic acid provides a model for Huntington's chorea, Nature, 263 (1976) 244-246.

13 Coyle, J.T., Molliver, M.E. and Kuhar, M.J., In situ injection of kainic acid: a new method for selectively lesioning neuronal cell bodies while sparing axons of passage, J. Comp. Neurol., 180 (1978) 301-324.

14 Crossman, A.R., Mitchell, I.J., Sambrook, M.A. and Jackson, A., Chorea and myoclonus in the monkey induced by gammaaminobutyric acid antagonism in the lentiform complex, Brain, 111 (1988) 1211-1233.

15 Crossman, A.R., Sambrook, M.A. and Jackson, A., Experimental hemichorea/hemiballismus in the monkey: studies on the intracerebral site of action in a drug-induced dyskinesia, Brain, 107 (1984) 479-596.

16 Curtis, D.R., Duggan, A.W., Felix, D., Johnston, G.A.R. and McLennan, H., Antagonism between bicuculline and GABA in the cat, Brain Research, 33 (1971) 57-73.

17 Curtis, D.R., Felix, D. and McLennan, H., GABA and hippocampal inhibition, Br. J. Pharmacol., 40 (1970) 881-883.

18 Davies, J. and Dray, A., Substance $P$ in the substantia nigra, Brain Research, 107 (1976) 623-627.

19 Dayhoff, J.E. and Gerstein, G.L., Favored patterns in spike trains, II. Application, J. Neurophysiol., 49 (1983) 1349-1363.

20 DeFelipe, J., Conti, F., Van-Eyck, S.L. and Manzoni, T., Demonstration of glutamate-positive axon terminals forming asymmetric synapses in cat neocortex, Brain Research, 455 (1988) 162-165.

21 DeLong, M.R., Activity of pallidal neurons during movement, J. Neurophysiol., 34 (1971) 414-427.

22 DeLong, M.R., Crutcher, M.D. and Georgopoulos, A.P., Primate globus pallidus and subthalamic nucleus: functional organization, J. Neurophysiol., 53 (1985) 530-543.

23 Deschenes, M., Paradis, M., Roy, J.P. and Steriade, M., Electrophysiology of neurons of lateral thalamic nuclei in cat: resting properties and burst discharges, J. Neurophysiol., 51 (1984) 1196-1219.

24 Domich, L., Oakson, G. and Steriade, M., Thalamic burst patterns in the naturally sleeping cat: a comparison between cortically projecting and reticularis neurones, J. Physiol. (London), 379 (1986) 429-449.

25 Eguchi, K. and Satoh, T., Relationship between positive sharp wave bursts and unitary discharges in the cat hippocampus during slow wave sleep, Physiol. Behav., 40 (1987) 497-509.

26 Fillion, M., Effects of interruption of the nigrostriatal pathway and of dopaminergic agents on the spontaneous activity of globus pallidus neurons in the awake monkey, Brain Research, 178 (1979) 425-441.

27 Fonnum, F., Gottesfeld, Z. and Grofová, I., Distribution of glutamate decarboxylase, choline acetyltransferase and aromatic amino acid decarboxylase in the basal ganglia of normal and operated rats. Evidence for striatopallidal, striatopeduncular and striatonigral GABAergic fibers, Brain Research, 143 (1978) 125-138.

28 Garcia-Rill, E., Hull, C.D., Levine, M.S. and Buchwald, N.A., The spontaneous firing patterns of forebrain neurons, IV. Effects of bilateral and unilateral frontal cortical ablation, Brain Research, 165 (1979) 23-36.

29 Greenamyre, J.T., Penney, J.B. and Young, A.B., Quantitative autoradiographic distribution of $\mathrm{L}-\left[{ }^{3} \mathrm{H}\right]$ glutamate-binding sites in rat central nervous system, J. Neurosci., 4 (1984) 2133-2144.

30 Groenewegen, H.J. and Russchen, F.T., Organization of the efferent projections of the nucleus accumbens to pallidal, hypothalamic, and mesencephalic structures: a tracing and immunohistochemical study in the cat, J. Comp. Neurol., 223 (1984) 347-367.

31 Hagihara, K., Tsumoto, T., Sato, H. and Hata, Y., Actions of excitatory amino acid antagonists on geniculo-cortical transmission in the cat's visual cortex, Exp. Brain Res., 69 (1988) $407-416$.

32 Jahnsen, H. and Llinás, R., Electrophysiological properties of guinea-pig thalamic neurones: an in vitro study, J. Physiol. (London), 349 (1984) 205-226.

33 Kita, H., Chang, H.T. and Kitai, S.T., Pallidal inputs to subthalamus: intracellular analysis, Brain Research, 264 (1983) 255-265.

34 Korn, S.J., Giachino, J.L., Chamberlin, N.L. and Dingledine, R., Epileptiform burst activity induced by potassium in the hippocampus and its regulation by GABA-mediated inhibition, J. Neurophysiol., 57 (1987) 325-340.

35 Legendy, C.R. and Salcman, M., Bursts and recurrences of bursts in the spike trains of spontaneously active striate cortex neurons, J. Neurophysiol., 53 (1985) 926-939.

36 Levine, M.S., Hull, C.D., Villablanca, J.R., Buchwald, N.A. and Garcia-Rill, E., Effects of caudate nuclear or frontal cortical ablation in neonatal kittens or adult cats on the spontaneous firing of forebrain neurons, Dev. Brain Res., 4 (1982) 129-138.

37 Monaghan, D.T and Cotman, C.W., Distribution of $n$-methylD-aspartate-sensitive L- $\left[{ }^{3} \mathrm{H}\right]$-glutamate-binding sites in rat brain, J. Neurosci., 5 (1985) 2902-2919.

38 Moriizumi, T., Nakamura, Y., Okoyama, S. and Kitao, Y., Synaptic organization of the cat entopeduncular nucleus with special reference to the relationship between the afferents to entopedunculothalamic projection neurons: an electron microscope study by a combined degeneration and horseradish peroxidase tracing technique, Neuroscience, 20 (1987) 797-816.

39 Nakanishi, H., Kita, H. and Kitai, S.T., Intracellular study of rat substantia nigra pars reticulata neurons in an in vitro slice preparation: electrical membrane properties and response characteristics to subthalamic stimulation, Brain Research, 437 (1987) 45-55.

40 Napier, T.C., Pirch, J.H. and Peterson, S.L., Spontaneous unit activity in the globus pallidus following cumulative injections of morphine in phenobarbital or chloral hydrate-anesthetized rats, Neuropharmacology, 22 (1983) 165-171.

41 Napier, T.C., Pirch, J.H. and Strahlendorf, H.K., Naloxone antagonizes striatally-induced suppression of globus pallidus unit activity, Neuroscience, 9 (1983) 53-59.

42 Obata, K. and Yoshida, M., Caudate-evoked inhibition and actions of GABA and other substances on cat pallidal neurons, Brain Research, 64 (1973) 455-459.

43 Park, M.R., Falls, W.M. and Kitai, S.T., An intracellular HRP study of the rat globus pallidus, I. Responses and light microscopic analyses, J. Comp. Neurol, 211 (1982) 284-294.

44 Penney, J.B. and Young, A.B., Striatal inhomogeneities and basal ganglia function, Movement Disorders, 1 (1986) 3-15.

45 Penney, J.B. and Young, A.B., Speculations on the functional anatomy of basal ganglia disorders, Annu. Rev. Neurosci., 6 (1983) 73-94.

46 Penny, G.R., Afsharpour, S. and Kitai, S.T., The glutamate 
decarboxylase-, leucine enkephalin-, methionine enkephalinand substance P-immunoreactive neurons in the neostriatum of the rat and cat: evidence for partial population overlap, Neuroscience, 17 (1986) 1011-1045.

47 Pinnock, R.D., Woodruff, G.N. and Turnbull, M.J., Actions of substance $\mathrm{P}, \mathrm{MIF}, \mathrm{TRH}$ and related peptides in the substantia nigra, caudate nucleus and nucleus accumbens, Neuropharmacology, 22 (1983) 687-696.

48 Ribak, C.E., Vaughn, J.E. and Roberts, E., The GABA neurons and their axon terminals in the rat corpus striatum as demonstrated by GAD immunocytochemistry, J. Comp. Neurol., 187 (1979) 261-284.

49 Robertson, R.G., Farmery, S.M., Sambrook, M.A. and Crossman, A.R., Dyskinesia in the primate following injection of an excitatory amino acid antagonist into the medial segment of the globus pallidus, Brain Research, 476 (1989) 317-322.

50 Sachdev, R.N.S., Gilman, S. and Aldridge, J.W., Effects of excitotoxic striatal lesions on single unit activity in globus pallidus and entopeduncular nucleus of the cat, Brain Research, 501 (1989) 295-306.

51 Sachdev, R.N.S. and Aldridge, J.W., Effect of striatal lesions on spontaneous unit activity recorded from the globus pallidus, entopeduncular nucleus, and ventral pallidum, Soc. Neurosci. Abstr., 13 (1987) 981.

52 Schwartzkroin, P.A. and Prince, D.A., Penicillin-induced activity in the hippocampal in vitro preparation, Ann. Neurol., 1 (1977) 463-466

53 Sirinathsinghji, D.J.S., Dunnett, S.B., Isacson, O., Clarke,
D.J., Kendrick, K. and Björklund, A., Striatal grafts in rats with unilateral neostriatal lesions, II. In vivo monitoring of GABA release in globus pallidus and substantia nigra, Neuroscience, 24 (1988) 803-811.

54 Smith, Y. and Parent, A., Neurons of the subthalamic nucleus in primates display glutamate but not GABA immunoreactivity, Brain Research, 453 (1988) 353-356.

55 Stringer, J.L. and Lothman, E.W., NMDA receptor dependent paroxysmal discharges in the dentate gyrus, Neurosci. Lett., 92 (1988) 69-75

56 Thompson, A.M., Inhibitory postsynaptic potentials evoked in thalamic neurons by stimulation of the reticularis nucleus evoke slow spikes in isolated rat brain slices, I, Neuroscience, 25 (1988) 491-502.

57 Thompson, A.M., Biphasic responses of thalamic neurons to GABA in isolated rat brain slices, II, Neuroscience, 25 (1988) 503-512.

58 Thompson, P.D., Berardelli, A., Rothwell, J.C., Day, B.L., Dick, J.P.R., Benecke, R. and Marsden, C.D., The coexistence of bradykinesia and chorea in Huntington's disease and its implications for theories of basal ganglia control of movement, Brain, 111 (1988) 223-244.

59 Vibert, J.F. and Costa, J., Spike separation in multiunit records: a multivariate analysis of spike descriptive parameters, Electroenceph. Clin. Neurophysiol., 47 (1979) 172-182.

60 Zaczek, R. and Coyle, J.T., Excitatory amino acid analogues: neurotoxicity and seizures, Neuropharmacology, 21 (1982) 1526. 